\title{
The Inhibitor Evaluation for Water Injection Pipeline of YanChang Oilfield
}

$$
\text { Xu Shiqii, a, Hao Shangjing }{ }^{2, b} \text { and Lisa }{ }^{3, c}
$$

${ }^{1}$ Shaanxi Key Laboratory of Advanced Stimulation Technology for Oil \& Gas Reservoirs, College of Petroleum Engineering, Xi'An ShiYou University, Xi'An,710065, China

2 School of Science, Xi'An ShiYou University, Xi'An,710065, China

\author{
School of Engineering, Technology and Maritime Operations, Liverpool John Moores University, \\ Liverpool, UK \\ acdlgong2000@163.com, b418902514@qq.com cb.li@ljmu.ac.uk
}

\begin{abstract}
Keywords: Corrosion inhibitor, Inhibitive efficiency, Corrosion mechanism, Water injection pipeline, Oilfield
\end{abstract}

Abstract. The main corrosion reason of water injection pipeline in YanChang oilfield is carbonate ions (or carbon dioxide gas) combined action of salts. The aim of the paper is to study the corrosion reason of the water injection pipeline in YanChang oilfield in order to develop an effective corrosion inhibitor for the water injection system. The corrosion inhibitor CQ-HO2 is synthesized by imidazoline introduced ethoxy groups. The evaluation of inhibition mechanism and inhibitive efficiency experiments were tested by weight-loss and electrochemical methods. The results of experiments show that the corrosion inhibitor CQ-HO2 is the anode type inhibitor, which can prevent the injection water pipeline from corrosion evidently. When the optimal concentration is $0.05 \%$ (weight per cent) at the temperature of $55^{\circ} \mathrm{C}$, the inhibitive efficiency is up to $88.1 \%$, which can mitigate the injection water pipeline corrosion of YanChang oilfield effectively.

\section{Introduction}

Water injection can fully complement formation energy, improve crude oil production and recovery factor, and make great contributions to the normal production of oil ${ }^{[1]}$. As the produced liquid water from the oilfield is increased day by day, the water re-injection scale is accumulated gradually, and the service life of pipeline networks has become longer and longer, so the corrosion problems of water injection system will be very prominent in recent years ${ }^{[2]}$. In order to ensure the safety and of operation in the water injection pipeline, it is urgent to take effective measures for internal corrosion protection.

The corrosion inhibitor is often used to slow down the corrosion in oilfield, because the corrosion inhibitor has these advantages of few injection dosage, excellent inhibition effect with environment friently, simple operation, low investment of the equipment and so on ${ }^{[3]}$. The protection effect of corrosion inhibitor is affected by corrosive medium, temperature, corrosion inhibitor dosage, metal materials and other factors ${ }^{[4]}$. That is, the selection of corrosion inhibitor is strict. Currently the adsorption of membrane-type inhibitors is used in oilfields mainly including the imidazoline kind, the amine and ammonium salts, sulfourea, organic acids, and others ${ }^{[5]}$.

YanChang oilfield is located in ShaanXi province of China. The main corrosion reason of water injection pipeline is the combination of carbonate ions (or carbon dioxide gas) and salts. In view of this characteristic, the imidazoline corrosion inhibitor CQ - HO2 introduced ethoxy groups was developed. The inhibitive effect of the corrosion inhibitor was evaluated by weight-loss method and electrochemical method.

\section{Materials and Methods}

A sample of NO 20 pipeline steel was obtained from YanChang oilfield. Its chemical components are shown in table 1. 
Table1 NO 20 pipeline steel components (\%)

\begin{tabular}{|c|c|c|c|c|c|c|c|c|}
\hline Pipeline steel & \multicolumn{7}{|c|}{ Chemical composition (weight per cent) } \\
\cline { 2 - 9 } & $\mathrm{c}$ & $\mathrm{Mn}$ & $\mathrm{Si}$ & $\mathrm{p}$ & $\mathrm{s}$ & $\mathrm{Ni}$ & $\mathrm{Cr}$ & $\mathrm{Cu}$ \\
\hline NO 20 & 0.20 & 0.45 & 0.18 & 0.03 & 0.025 & 0.20 & 0.20 & 0.23 \\
\hline
\end{tabular}

The sample was cut into the size of $25 \mathrm{~mm} \times 10 \mathrm{~mm} \times 2 \mathrm{~mm}$ to observe corrosion morphology and the size of $10 \mathrm{~mm} \times 10 \mathrm{~mm} \times 2 \mathrm{~mm}$ to have electrochemical experiments. Samples processed in accordance with the requirements for processing, washed by distilled water and acetone to remove oil and dust of sample, and then washed by the anhydrous ethanol, the sample was blew by cold wind, finally put the sample into the prepared dry vessel for experiments ${ }^{[6]}$. The experiments were carried out according to oil and gas industry standard SY/T5273-2000 evaluation method for behaviour of corrosion inhibitor in produced water from the oilfield.

The experimental medium is injection water, which is produced water after treatment from YanChang oilfield. The sample was taken at the standard sampling point and the composition and concentration of ions have been tested by ICP-OES. All kinds of ion content are shown in table 2, and the $\mathrm{PH}$ is 8.2 .

Table2 The each ion content of experimental medium

\begin{tabular}{|c|c|c|c|}
\hline Ion & content $(\mathrm{mg} / \mathrm{L})$ & Ion & content $(\mathrm{mg} / \mathrm{L})$ \\
\hline $\mathrm{K}^{+}, \mathrm{Na}^{+}$ & 416.1 & $\mathrm{Cl}^{-}$ & 592.0 \\
\hline $\mathrm{Ca}^{2+}$ & 60.1 & $\mathrm{SO}_{4}^{2-}$ & 206.5 \\
\hline $\mathrm{Mg}^{2+}$ & 52.3 & $\mathrm{HCO}_{3}{ }^{-}$ & 268.5 \\
\hline $\mathrm{NH}_{4}^{+}$ & 0.16 & $\mathrm{CO}_{3}{ }^{2-}$ & 0.0 \\
\hline \multicolumn{2}{|c|}{ Total salinity } & & 1595.7 \\
\hline
\end{tabular}

Electrochemical experiment equipment was adopted M2273 electrochemical testing system with the three electrodes system manufactured by the United States. The working electrode was NO 20 pipeline steel, reference electrode was saturated calomel electrode, and auxiliary electrode was platinum electrode. The polarization curves and impedance spectra of pipeline steel were tested under different concentrations of $\mathrm{CQ}-\mathrm{HO} 2$ corrosion inhibitors, the scanning speed was $0.5 \mathrm{mV} / \mathrm{s}$, the temperature was controlled at $55^{\circ} \mathrm{C}$ and a $10 \mathrm{mV}$ perturbation signal was applied over a frequency range from $5 \mathrm{mHz}$ to $100 \mathrm{kHz}$. The Zsimpwin software was used to analyse AC impedance spectrum .

\section{Results and Discussion}

The experimental temperature was $55^{\circ} \mathrm{C}$ (It is injection water temperature of YanChang oilfield), Corrosion inhibitor concentration was all weight percentage.

The first evaluation experiment of corrosion inhibitor CQ- HO2 was used the weight-loss method. Experimental time was 72 hours. Three parallel experiments were conducted at the same time, these inappropriate experimental data was removed and then the corrosion rates were averaged. The relationship between the inhibitor CQ - HO2 concentration and the inhibition efficiency was shown in figure 1. 


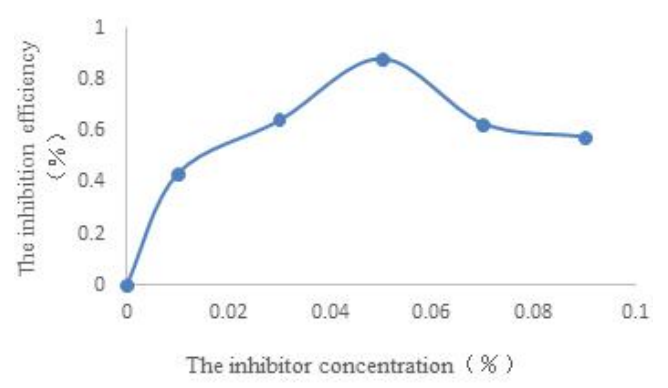

Figure 1 The relationship between the inhibitor CQ - HO2 concentration and the inhibition efficiency

The relationship between inhibitor CQ-HO2 concentration and the inhibition efficiency showed that: When the inhibitor concentration is less than $0.05 \%$, with the rising of corrosion inhibitor CQ-HO2 concentration, the inhibition efficiency increased gradually, and the corrosion rate slowed down; However when the corrosion inhibitor CQ-HO2 concentration was above $0.05 \%$, the corrosion efficiency decreased again. When corrosion inhibitor CQ-HO2 concentration was $0.05 \%$, the corrosion inhibition efficiency was as high as $88.1 \%$.

The second evaluation experiment of corrosion inhibitor CQ - HO2 was the electrochemical method The scanning range for the Tafle polarization cures was set at $-300 \mathrm{mV} \sim 300 \mathrm{mV}$. The working electrode surface area was $1 \mathrm{~cm}^{2}$, So current and current density had the same changing trend. These Polarization curves under different CQ - HO2 corrosion inhibitor concentration were shown in Figure 2 .

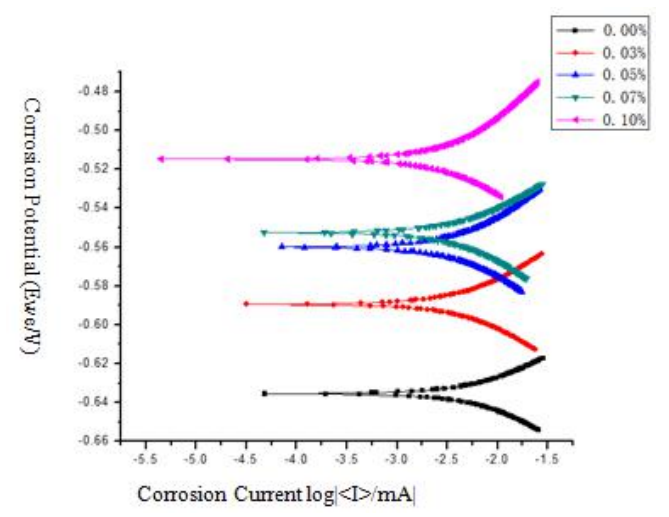

Figure 2 The Polarization curve under different CQ - HO2 corrosion inhibitor concentration

It was found that the corrosion potential of the polarization curve gradually moved to positive direction with the rising of corrosion inhibitor CQ-HO2 concentration, but corrosion current density of cathode and anode gradually shift to the left. With the development of corrosion process, the inhibition of cathode and anode was strengthened. Comparing the cathode polarization curve slope without corrosion inhibitor, the slope increased firstly, and then declined. The steeper the slope, the higher the polarization resistance value. The reduction of polarization current accounted for the cathode reaction which was restrained. On the contrary, the decreased slope accounted for the decline in the polarization resistance ${ }^{[7]}$. The inhibition of cathode reaction was weakened. The reason was that inhibitor molecules were adsorbed on the electrode surface at the beginning or corrosion product film decreased the corrosion of metals, while with the increase of potential, the adsorbed corrosion inhibitor on electrode surface began to desorb ${ }^{[8]}$. The anode had a more obvious change than the cathode did, so the corrosion inhibitor belonged to the anode corrosion inhibitor. According to polarization curve, the experimental results were consistent with the result of weight-loss experiment.

The range of electrochemical impedance spectroscopy frequency was $5 \mathrm{mHZ} 100 \mathrm{kHZ}$ of the corrosion inhibitor CQ-HO2 evaluation by AC impedance spectroscopy. The measured Nyquist diagram under different concentration inhibitor was shown in figure 3. 


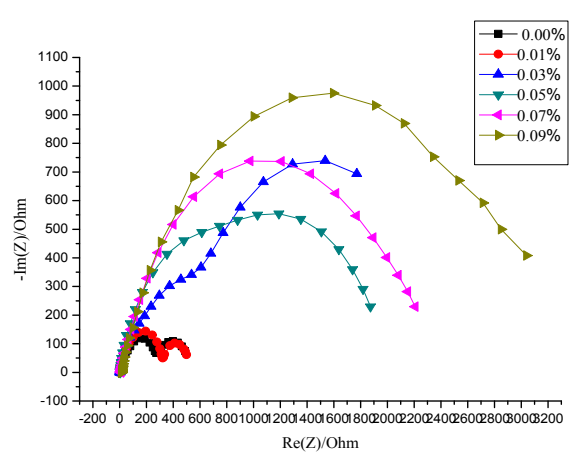

Figure 3 Nyquist figure under different corrosion inhibitor CQ-HO2 concentration

Electrochemical parameters were obtained from the EIS method. These EIS of the corrosion inhibitor concentrations were $0.01 \%$ and $0.09 \%$ which could be found in Figure 4(a), and the EIS of other concentration could be founded in Figure $4(b)$. In the Figure, $R_{s}$ was solution resistance, $R_{p 1}$ and $R_{p 2}$ were polarization resistances, $R_{t}$ was charge-transfer resistance, $C_{1}$ and $C_{2}$ were the electric double layer capacitances between the electrodes and the dispersed phase, and electric double layer capacitors were $\mathrm{Q}, \mathrm{Q}_{1}$ and $\mathrm{Q}_{2}$.

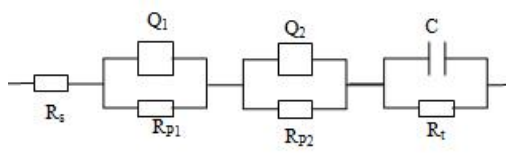

(a)

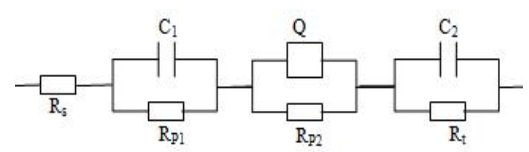

(b)

Figure 4 The equivalent circuit diagram

(a) Corrosion inhibitor concentrations were $0.01 \%$ and $0.09 \%$

(b)Corrosion inhibitor concentrations were $0.00 \%, 0.03 \%, 0.05 \%$ and $0.07 \%$

The different concentrations of Nyquist figure showed that the impedance diagram had two constants corresponding to the high-frequency capacitive reactance arc and the low-frequency capacitive reactance arc. The high-frequency capacitive reactance arc related to the-electric double layer capacitance of the interface and the solution resistance, what described the chemical reaction ${ }^{[9]}$. When the inhibitor concentration was $0.01 \%$, its trend in Figure 4 was similar as the Nyquist figure without corrosion inhibitor. Thus, we can draw a conclusion that the effect of inhibition was not obvious when the corrosion inhibitor was less than $0.01 \%$. With the increase of corrosion inhibitor concentration, the capacitive reactance radius of the high-frequency area increased gradually. With the increase of the corrosion inhibitor concentration, the effect of the corrosion-inhibition was gradually obvious. Low-frequency capacitive reactance arc related to the adsorption intermediate showed on the nature of the interface ${ }^{[10]}$. Curve fitting results showed that the solution resistance Rs(from 0.9987

$\Omega{ }^{\cdot} \mathrm{cm}^{2}$ to12.08 $\Omega{ }^{\cdot} \mathrm{cm}^{2}$ ) and the charge transfer resistance Rt (from54.2 $\Omega{ }^{\circ} \mathrm{cm}^{2}$ to $343.5 \Omega{ }^{\circ}$ $\mathrm{cm}^{2}$ )gradually rose as corrosion inhibitor concentration increased. In other words, the ability to block the charge movement was enhanced. the corrosion current decreased, and the corrosion reaction declined. When the corrosion inhibitor concentration was higher than the optimal concentration $0.05 \%$, the charge transfer resistance $\operatorname{Rt}\left(\right.$ from $67.6 \Omega{ }^{\cdot} \mathrm{cm}^{2}$ to $20.87 \Omega{ }^{\cdot} \mathrm{cm}^{2}$ ) decreased again, so the corrosion rate increased because of the corrosion inhibitor desorption ${ }^{[11]}$.

The experiment results showed that the inhibition effect of corrosion inhibitor CQ-HO2 was obvious at the temperature $55^{\circ} \mathrm{C}$ within a certain concentration range. The adsorption film effect was more obvious on the specimen surface, which could restrain the diffusion of ions and charges on the surface of the specimen. Under this situation, the film prevented the electrochemical reaction, and that was the reason why the rate of corrosion reaction reduced gradually. 


\section{Conclusion}

-The corrosion inhibitor CQ-HO2 was synthesized by imidazoline introduced ethoxy groups, which can inhibit the injection water pipeline of YanChang oilfield from corrosion efficiently.

- The optimum concentration of forming film of corrosion inhibitor CQ-HO2 was $0.05 \%$ under the temperature $55^{\circ} \mathrm{C}$, where the inhibitive efficiency is up to $88.1 \%$.

- The corrosion mechanism of the corrosion inhibitor CQ-HO2 was studied by the electrochemical experiment. The corrosion inhibitor belonged to the type of adsorption and anode corrosion inhibitor. The kind of corrosion inhibitor could prevent the corrosion of injection pipeline efficiently when the injection water included amounts of carbonate ions (or carbon dioxide gas) in salt solution combined action of salts.

\section{Acknowledgements}

This work was financially supported by Dr scientific research project (Project NO. YS2903200138).

\section{Reference:}

[1]Jiang, G., Hu, D., Ji, C.F. and Song, Y.G. The research of corrosion mechanism and protection measure of water-flood pipes. TT 2009: Advances and Experiences with pipelines and Trenchless Technology for Water, Sewer, Gas, and Oil Applications, ASCE, Reston, VA, pp.508-520.

[2] Ogundele, G. I., White, W. E. Some observation on corrosion of carbon steel in aqueous environment containing carbon dioxide. Corrosion, 42(2), 71-76(1986).

[3]Jiang, D. L., Cheng, D.W., et al. Corrosion analysis, and use of an inhibitor in oil well. Chem Intermed, 40, 649-660(2014).

[4]Hong, T., Sun, Y. H., Jepson, W. P. Study on corrosion inhibitor in large pipelines under multiphase flow using EIS. Corros. Sci., 44, 101-112(2002).

[5]Chen, G., Su, H., Song et al. Synthesis and evaluation of isatin derivatives as corrosion inhibitors for Q235A steel in highly concentrated HCl. Research on Chemical Intermediates, October 2012, pp.1-10.

[6]Cao, C. N. On the impedance plane displays for irreversible electrode reactions based on the stability conditions of ready state-I. One state variable besides electrode potential, Electrochemical Acta, 35(5), 831-836(1990) .

[7]Dong, L., Liu, H. Q., et al. Corrosion of water injection system in Shengli oil field. Anti-Corrosion Methods and Materials, 60(4),185-193(2013).

[8]Hamid, Z.A., Aal, A.A. effect of electroless process parameters for nickel-phosphorous coatings on carbon fibres. Anti-corrosion methods and Materials, 57, 173-179(2010) .

[9]Ramachandran, S., Campbell, S., Ward, M.B. Interactions and properties of corrosion inhibitors with by product layers. Corrosion, 57(6),508-515(2001) .

[10]Chen, Y.,Jepson, W.P. EIS measurement for corrosion monitoring under multiphase flow conditions. Electrochemical Acta, 44(24), 4453-64(1999) .

[11]Ho, C.H., Raistrick, D. and Huggins, R. A. Application of AC techniques to study of lithium diffusion in tungsten trioxide thin films. Journal of the Electrochemical Society, 127(2), 345-50(1980). 